\title{
Frailty assessment in very old intensive care patients: the Hospital Frailty Risk Score answers another question
}

\author{
Raphael Romano Bruno', Bertrand Guidet ${ }^{2,3,4}$, Bernhard Wernly ${ }^{5,6}$, Hans Flaatten ${ }^{7,8}$ and Christian Jung ${ }^{*}$ (D)
}

(c) 2020 The Author(s)

\section{Dear Editor,}

We read with great interest the recently published letter by Redfern et al. [1] commenting on our article on frailty in Intensive Care Medicine [2]. Redfern et al. performed a retrospective analysis in 31,812 patients (aged 75 years and older) and showed that the Hospital Frailty Risk Score (HFRS) reflects well the probability of unplanned intensive care unit (ICU) admission. Briefly, the HFRS estimates a patient's frailty on the basis of electronically available data on chronic ICD-10 diagnoses assigned to the patient [3].

In contrast, the Clinical Frailty Scale (CFS) used in Guidet et al. [2] is based on a different concept. The WHO defines frailty as a clinically identifiable condition in which the ability of older people to cope with everyday and acute stressors is reduced [4]. The main reason for this reduced physiological functional reserve depends not only on age and diagnoses, but also on genetics, epigenetics, and environmental-even social-factors. These different factors lead, via cumulative molecular and cellular damage, to the reduced physiological reserve that can affect all organ systems. Therefore, the assessment of frailty should incorporate this multidimensional concept of "intrinsic capacity".

Nowadays, two key aspects characterize triage in daily intensive care medicine. First, decision-making in this setting is often time-critical. Second, in these times of limited ICU capacities, this decision-making can decide

\footnotetext{
*Correspondence: Christian.Jung@med.uni-duesseldorf.de ${ }^{1}$ Department of Cardiology, Pulmonary Diseases, and Vascular Medicine, Medical Faculty, Heinrich Heine University of Duesseldorf, Duesseldorf, Germany

Full author information is available at the end of the article
}

the survival or death of a patient and must therefore be based on the best available knowledge.

With regard to the first aspect, the CFS can be administered very quickly and by health care providers of different professional backgrounds without loss of reliability. The CFS showed very good interrater agreement and very little missing information, suggesting that this tool is reliable and easy to use [2].

In contrast, the HFRS relies on patient records, which are prone to be incomplete or possibly incorrect. ICD codes cannot reflect disease severity; they are normally used for reimbursement purposes.

The result of the assessment should help to predict the patient's clinical course. Redfern et al. confirmed previously published studies showing that the HFRS does not predict mortality in critically ill patients [5]. On the contrary, the CFS has been shown to estimate intra-hospital survival [2]. Of course, the information provided by the HFRS on the likelihood of ICU readmission is of some importance. Still, its ability to provide this information is not surprising given that counting ICD codes correlates with the overall grade of morbidity, which can be considered a sub-dimension of frailty. Thus, compared with the CFS, the HFRS captures a different-smaller-dimension of frailty.

Triage decisions in intensive care medicine must be quick and reliable. Ideally, we asses our patients in multiple dimensions. Frailty research is still in its infancy, but the CFS seems to be a rapid and multidimensional tool. The HFRS, on the other hand, is less accurate and prone to errors as it answers another question.

\section{勿




\section{Author details}

1 Department of Cardiology, Pulmonary Diseases, and Vascular Medicine, Medical Faculty, Heinrich Heine University of Duesseldorf, Duesseldorf, Germany. ${ }^{2}$ Assistance Publique - Hôpitaux de Paris, Hôpital Saint-Antoine, Service de Réanimation Médicale, 75012 Paris, France. ${ }^{3}$ UPMC Univ Paris 06, UMR_S 1136, Institut Pierre Louis d'Epidémiologie et de Santé Publique, Sorbonne Universités, 75013 Paris, France. ${ }^{4}$ INSERM, UMR_S 1136, Institut Pierre Louis d'Epidémiologie et de Santé Publique, 75013 Paris, France. ${ }^{5}$ Department of Cardiology, Paracelsus Medical University, Salzburg, Austria. ${ }^{6}$ Division of Cardiology, Department of Medicine, Karolinska Institutet, Karolinska University Hospital, Stockholm, Sweden. ${ }^{7}$ Department of Clinical Medicine, University of Bergen, Bergen, Norway. ${ }^{8}$ Department of Anaestesia and Intensive Care, Haukeland University Hospital, Bergen, Norway.

\section{Acknowledgements}

Open Access funding provided by Projekt DEAL.

\section{Compliance with ethical standards}

\section{Conflicts of interest}

The authors whose names are listed immediately above certify that they have no affiliations with or involvement in any organization or entity with any financial interest (such as honoraria; educational grants; participation in speakers' bureaus; membership, employment, consultancies, stock ownership, or other equity interest; and expert testimony or patent-licensing arrangements), or non-financial interest (such as personal or professional relationships, affiliations, knowledge or beliefs) in the subject.

\section{Open Access}

This article is licensed under a Creative Commons Attribution-NonCommercial 4.0 International License, which permits any non-commercial use, sharing, adaptation, distribution and reproduction in any medium or format, as long as you give appropriate credit to the original author(s) and the source, provide a link to the Creative Commons licence, and indicate if changes were made. The images or other third party material in this article are included in the article's Creative Commons licence, unless indicated otherwise in a credit line to the material. If material is not included in the article's Creative Commons licence and your intended use is not permitted by statutory regulation or exceeds the permitted use, you will need to obtain permission directly from the copyright holder. To view a copy of this licence, visit http://creativecommons.org/licen ses/by-nc/4.0/.

\section{Publisher's Note}

Springer Nature remains neutral with regard to jurisdictional claims in published maps and institutional affiliations.

\author{
Accepted: 8 May 2020 \\ Published online: 25 May 2020
}

\section{References}

1. Redfern OC, Harford M, Gerry S, Prytherch D, Watkinson PJ (2020) Frailty and unplanned admissions to the intensive care unit: a retrospective cohort study in the UK. Intensive Care Med. https://doi.org/10.1007/ s00134-020-06020-7

2. Guidet B, de Lange DW, Boumendil A, Leaver S, Watson X, Boulanger C, SzczeklikW, Artigas A, Morandi A, Andersen F, Zafeiridis T, Jung C, Moreno R, Walther S, Oeyen S, Schefold JC, Cecconi M, Marsh B, Joannidis M, Nalapko Y, Elhadi M, Fjolner J, Flaatten H, Group VIPs (2020) The contribution of frailty, cognition, activity of daily life and comorbidities on outcome in acutely admitted patients over 80 years in European ICUs: the VIP2 study. Intensive Care Med 46:57-69

3. Gilbert T, Neuburger J, Kraindler J, Keeble E, Smith P, Ariti C, Arora S, Street A, Parker S, Roberts HC, Bardsley M, Conroy S (2018) Development and validation of a hospital frailty risk score focusing on older people in acute care settings using electronic hospital records: an observational study. Lancet 391:1775-1782

4. Clegg A, Young J, liffe S, Rikkert MO, Rockwood K (2013) Frailty in elderly people. Lancet 381:752-762

5. Bruno RR, Wernly B, Flaatten H, Scholzel F, Kelm M, Jung C (2019) The hospital frailty risk score is of limited value in intensive care unit patients. Crit Care 23:239 